\title{
THE OUTCOMES OF ARTHROCENTESIS WITH HIGH PRESSURE VERSUS LOW PRESSURE IN TEMPOROMANDIBULAR JOINT DISORDERS
}

\author{
Ahmed M. Attia*, Ahmed M. Adawy**
}

\begin{abstract}
Objective: This study was designed to evaluate the outcomes of arthrocentesis with high versus low pressure in temporomandibular joint disorders. Subjects and methods: The present study included 56 patients, 36 females and 20 males. Patients were selected from those attending oral and maxillofacial outpatient clinic of Sayed Galal university hospital. All were complaining of temporomandibular pain and seeking treatment. Data collected in form of chief complaint, duration of condition and history of complaint that included side, duration, onset, precipitating and relieving factors, and relation to other activities such as eating, or shouting. Also, the presence of parafunctional habits was recorded. Assessment of pain level was made using visual analogue scale. Measurement of maximum mouth opening, Orthopantogram was taken for all of them to rule out degenerative joint diseases. Magnetic resonance images (MRI) were taken for all patients for accurate diagnosis of joint condition, disc position and shape. Results: After 1,2,3 months, there was no statistically significant difference between pain scores in the two groups, after $1,2,3$ months, there was a statistically significant difference between MMO in the two groups, Low pressure arthrocentesis group showed statistically significantly lower mean MMO than high pressure arthrocentesis group. Conclusion: Pain reduction was noted in both groups Maximal mouth opening improved with high pressure arthrocentesis more than with low pressure arthrocentesis.
\end{abstract}

KEY WORDS: Arthrocentesis, Temporomandibular Joint Disorders

\section{INTRODUCTION}

Internal derangement of the temporomandibular joint is one of the most common forms of temporomandibular disorders. The term refers to clinical criteria classifying TMJ disorders but is generally used to denote a mechanical fault in the joint interfering with its smooth movement, such as abnormal positional relationship of the articular disc to the mandibular condyle and the articular eminence. The disorders have been associated with characteristic clinical findings, including pain, joint sounds, and irregular or deviated jaw functions ${ }^{(1)}$.

The first line of treatment in temporomandibular joint internal derangement is conservative treatment including behavior modification, physical therapy, medication, jaw exercises and intra-oral appliances. If the patient not responds to conservative treatment the second line of treatment is minimally invasive arthrocentesis ${ }^{(2)}$. Arthrocentesis of the temporomandibular joint first described by Nitzan et $\mathrm{al}^{(3)}$ as the simplest form of surgery in the TMJ, aiming to release the articular disc and to remove adhesions between the disc surface and the glenoid fossa.

Few studies evaluated the effect of low versus high pressure during arthrocentesis. Low pressure was effective for treating patients with acute closed lock of the temporomandibular(TMJ) without severe joint pain or bone changes. Arthrocentesis under high pressure removes adhesions and widens joint spaces it is highly efficient procedure for treating

\footnotetext{
* Dentist, Nasr City Insurance Hospital, Cairo.

** Professor, Department of Oral and Maxillofacial Surgery, Faculty of Dental Medicine, Boys, Cairo Al-Azhar University.
} 
patients with chronic closed lock and adhesions in upper joint space (4). The definition of "high" and "low" pressure, however, is lacking. Moreover, a device that permits constant pressure, without fluctuations is not available. The aim of this study was to evaluate the outcomes of arthrocentesis with high versus low pressure in temporomandibular joint disorders.

\section{SUBJECTS AND METHODS}

Patients were divided at random into two equal groups. Group I comprised 20 patients, 7 males and 13 females. Their ages ranged from 22 to 51 years, with a mean age values of 35.7 (SD8.4) years. Group II comprised 20 patients, 6 males and 14 females. Their age ranged from 18 to 50 years, with a mean age values of 34.9 (SD9.9) years.

Group 1: included 20 joints. Each joint underwent arthrocentesis using lactated Ringer's solution for Joint lavage under high pressure.

Group 2: included 20 joints. Each joint underwent arthrocentesis using lactated Ringer's solution for joint lavage under low pressure.

\section{A -Preoperative phase}

All patients underwent pre-operative clinical examination: Patients' data were collected; name, gender and age, medical and dental histories were taken. Assessment of pain level was made using visual analogue scale. Measurement of maximum mouth opening and palpation of masticatory muscles for trigger points were performed.

Dental examination for the condition of hard and soft tissues of the oral cavity was identified and recorded. Orthopantogram was taken for all of them to rule out degenerative joint diseases. Magnetic resonance images (MRI) were taken for all patients for accurate diagnosis of joint condition, disc position and shape.

\section{B -Operative phase}

Initially all patients were subjected to conservative treatment modalities for a period of 3 months. Arthrocentesis and joint lavage procedures were performed according to technique described by Nitzan ${ }^{(5)}$. The procedure was conducted under conscious sedation using combination of intravenous incremental dose of Phentanyl and Dormicum according to patient need. Patient preparation using Betadine Scrub solution. Sterile cotton soaked in antibiotic ointment was placed in the external auditory canal for protection of the ear from blood and fluid. The auriculotemporal nerve was blocked with about $2 \mathrm{ml}$ of local anesthetic (Mepecaine-L).

The entry point of the inflow cannula was marked $10 \mathrm{~mm}$ anterior to tragus and $2 \mathrm{~mm}$ below imaginary canthal-tragal line. The second point for the outflow cannula located $20 \mathrm{~mm}$ anterior to tragus and $8 \mathrm{~mm}$ below canto-tragal plane, as shown in. A sterile mouth opener was placed on dental arch contralateral to the arthrocentesis side to allow jaw head displacement downward and to the front, helping the approach to the posterior recess of upper TMJ compartment. A 20 gauge needle was inserted in the most posterior point, connected to a $5 \mathrm{ml}$ syringe where 1 to $4 \mathrm{ml}$ of the $0.9 \%$ saline solution was administered aiming at distending the joint space. Another needle was introduced in the distended compartment, in front of the first needle in the previously marked point. The first attempt for arthrocentesis under pressure was made using aneroid manometer gauge of aneroid sphygmomanometer. With such device, constant pressure during arthrocentesis was not maintained. The second attempt to fix the pressure used during arthrocentesis was made using infuser blood pressure C-Fuser containing a plastic cuff (figure 1). 


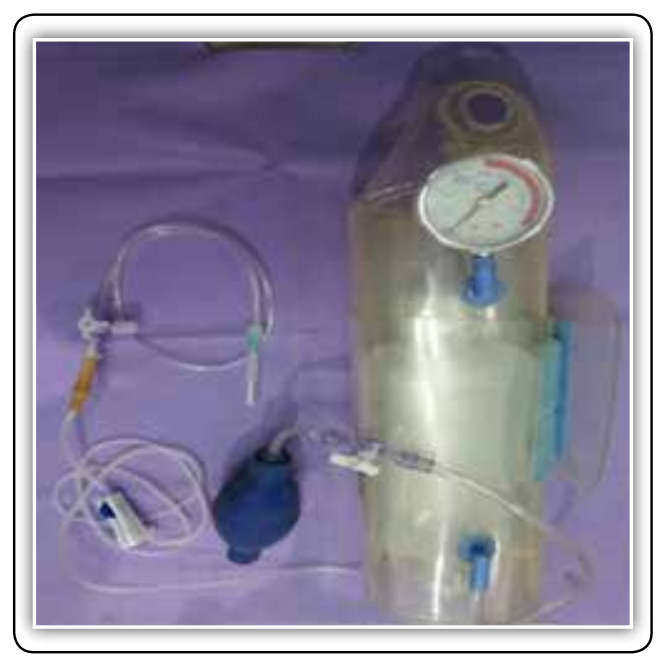

FIG (1) C-fuser containing lactated Ringer connected to three ways cannula by I.V. infusion set.

Lactated Ringer solution was placed in the cuff of the C-fuser, and the cuff was properly closed. The solution was connected to a three way cannula by I.V. infusion set which was connected to the posterior needle inserted in the 10-2 point marked previously on the patient. First pressing on the squeeze bulb of the c-fuser was performed to elevate the pressure. At low pressure $(150 \mathrm{mmHg})$ the outflow of the fluid through the second needle started slowly drop by drop as for lavage the joint with $250 \mathrm{ml}$ for 5 minutes. On the other hand, at high pressure (400 $\mathrm{mmHg}$ ) outlet of the fluid was continuous and rapid for lavage of the joint with $250 \mathrm{ml}$ for 3 minutes. Then the air release valve of the c-fuser was closed to fix the pressure. After that the three-way valve was opened to allow the flow of the fluid through the posterior needle. After lavage, the I.V. infusion set valve was closed, the outlet needle was removed, and $2 \mathrm{ml}$. of Hyalgan was injected in the joint cavity. After injection the inflow needle was removed.

\section{C-Postoperative phase}

Patients were instructed to apply ice packs 20 minutes each hour the same day of surgery.
Unasyn375mg tablets were given 3 times daily

Cataflam50mg was prescribed twice daily.

All subjects were followed up one month, three months, six months post operatively to assess their clinical conditions.

\section{D- Follow up phase}

1. Degree of joint pain using visual analogue scale.

2. Presence or absence of joint clicking and sounds.

3. Improvement of maximal mouth opening

Data were represented as mean and standard deviation. Repeated measures analysis of variance (ANOVA) test was used to compare numeric variables within the studied group of patients. Post Hoc test was made if ANOVA or Friedman tests were positive. Using SPSS version in all tests, result was considered statistically significant if the p- value was less than 0.05 .

\section{RESULTS}

\section{Pain Scores}

Comparison between the two groups After 1 month, there was no statistically significant difference between pain scores in the two groups with mean value $6 \pm 1.41$ in low pressure group and mean value $6.19 \pm 1.6$ in high pressure group. Three months after arthrocentesis, no significant difference between pain scores in the two groups was noticed with mean value $4.87 \pm 1.68$ in low pressure group and mean value $5 \pm 1.63$ in high pressure group. Even after 6 months, no significant difference was found between pain scores in the two groups with mean value $4.07 \pm 2.37$ in low pressure group and mean value $4.06 \pm 2.05$ in high pressure group

Maximum Mouth Opening (MMO)

Comparison between the two groups 
After one month, the mean value for the low pressure arthrocentesis group was $30.2 \pm 5.77 \mathrm{~mm}$ compared to $33.4 \pm 5.77 \mathrm{~mm}$ with high pressure arthrocentesis group. Three months after the operation, the mean value of low pressure group was $31.6 \pm 6.38 \mathrm{~mm}$ which was significantly lower to that of high pressure group $(35.7 \pm 2.03 \mathrm{~mm})$. The same trend was also calculated at 6 months following the operation. Low pressure arthrocentesis group showed statistically significantly lower mean value of maximal mouth opening $(32.4 \pm 7.83 \mathrm{~mm}$.) compared to $37.95 \pm 2.37 \mathrm{~mm}$. with high pressure arthrocentesis group.

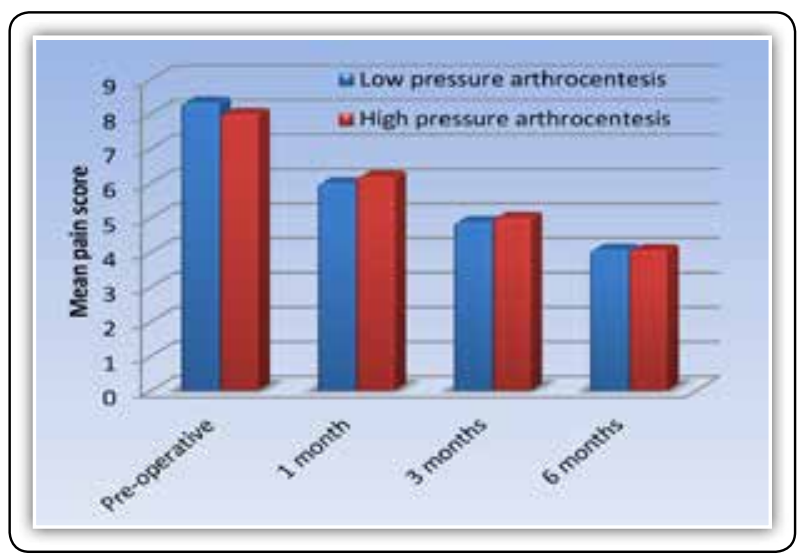

FIG (1) Bar chart representing mean pain scores in the two groups

MRI were taken 6 months after arthrocentesis after the procedure for documentation and for comparison with the preoperative ones. Which indicates no change in disc position after arthrocentesis with low and high pressure.

\section{DISCUSSION}

Temporomandibular disorders (TMD) are important oral health problems. It has been estimated that approximately $20 \%$ to $30 \%$ of the adult population will experience temporomandibular joints dysfunctions according to James Swift et $\mathrm{al}^{(6)}$. Reversible conservative therapy such as cognitive behavioral therapy, physical therapy, pharmacological therapy and intraoral appliances should be considered for the first-line management of TMD. If the patient dosn't responds to conservative treatment, the second line of treatment is the minimally invasive arthrocentesis. Nitzan et al ${ }^{(7)}$, Dimitroulis et al. ${ }^{(8)}$, Kaneyama et al. ${ }^{(9)}$, Sanroman et al. ${ }^{(10)}$. performed arthrocentesis under low pressure while Alkan and Kilic (11), Yura and Totsuka ${ }^{(12)}$ performed arthrocentesis under high pressure. At first, attempt for arthrocentesis under pressure was made using aneroid manometer gauge of aneroid sphygmomanometer.

In a second attempt Smith's Medical C-fusor Pressure Infuser's device with a plastic cuff was used. There was no statistically significant difference in pain score after arthrocentesis under high and low pressure. This finding indicated that, the pain reduction is due to irrigation which washes away inflammatory mediators and catabolic products and thus providing pain relief. Concerning maximal mouth opening, the results of the present study indicated that in both high and low pressure groups, significant improvement, compared to the preoperative values, was obtained in the immediate post-operative period, mouth opening further increased from third to sixth months. Significant differences, however, were observed between the two groups. The postoperative MRI findings revealed no change in the disc position for cases presented with anterior disc displacement without reduction. These findings are in full agreement with the results of Moses et al ${ }^{(13)}$ which indicated that $92 \%$ of the patients had persistent anterior disc displacement, even they had a significant reduction in pain and restoration of normal mandibular function, which is may be due to the retrodiscal tissue tends to become less elastic and more fibrous, which causes the reduction to occur more difficult.

\section{CONCLUSIONS}

Conservative treatment should be resorted first. 16 out of 56 patients improved with this treatment modality. Arthrocentesis is highly effective and 
all patients had a significant reduction in pain and restoration of normal mandibular function. Pain reduction was noted in both groups immediately after the procedure and maintained during 6 months after treatment. Maximal mouth opening improved with high pressure arthrocentesis more than with low pressure arthrocentesis. MRI findings indicated that there was no change in disc position even after improvement of mouth opening.

\section{REFERENCES}

1. Yucel MA, Gozneli R, Alkumru HN, Ozkan YK. Evaluating the additional effects of arthrocentesis on the condylar pathways of temporomandibular joint in patients with internal derangement treated with stabilizing splint.J Cran Maxillofac Surg. 2013;30 :1-5.

2. Madani AS, and Mirmortazavi A. Comparison of three treatment options for painful temporomandibular joint clicking. J of Oral Science. 2011; 53:349-54

3. Nitzan DW, Dolwick MF, and Martinez GA. Temporomandibular Joint Arthrocentesis: A Simplified Treatment for Severe, Limited Mouth Opening. J Oral Maxillofac Surg. 1991; 49:1163-7.

4. Yura S and Totsuka Y. Relationship Between Effectiveness of Arthrocentesis Under Sufficient Pressure and Conditions of the Temporomandibular Joint. J Oral Maxillofac Surg. 2005; 63:225-8.

5. Nitzan DW, Dolwick MF, and Martinez GA. Temporomandibular Joint Arthrocentesis: A Simplified Treatment for Severe, Limited Mouth Opening. J Oral Maxillofac Surg. 1991; 49:1163-7.
6. Swift JQ, Roszkowski MT, Alton T, Hargreaves KM. Effect of Intra-Articular versus systemic Anti-inflammatory Drugs in a Rabbit Model of Temporomandibular Joint Inflammation. J Oral Maxillofac Surg.1998; 56:1288-95.

7. Nitzan DW, Dolwick FM, and Martinez GA. Temporomandibular Joint Arthrocentesis: A Simplified Treatment for Severe, Limited Mouth Opening. J Oral Maxillofac Surg. 1991; 49:1163-7.

8. Dimitroulis G, Dolwick MF, Martinez A. Temporomandibular joint arthrocentesis and lavage for the treatment of closed lock: a follow-up study. Br J of Oral and Maxillofac Surg.1995; 33:23-7.

9. Kaneyama K, Natsuki S, Shinichi T, Fujimura K, Sato J, Nagao T. Anchored disc phenomenon with a normally positioned disc in the temporomandibular joint Characteristics and behavior. Br J of Oral and Maxillofac Surg. 2007; $45: 279-83$.

10. Sanroman JF. Closed lock (MRI fixed disc): a comparison of arthrocentesis and arthroscopy. Int J Oral Maxillofac. Surg. 2004; 33: 344-8.

11. Alkan A, Kilic E. Anew approach to arthrocentesis of the temporomandibular joint. Int J Oral Maxillofac. Surg. 2009; 38: 85-6.

12. Yura S and Totsuka Y. Relationship Between Effectiveness of Arthrocentesis Under Sufficient Pressure and Conditions of the Temporomandibular Joint. J Oral Maxillofac Surg. 2005; 63:225-8.

13. Moses JJ, Sartoris D, Glass R, Tanaka T, Poker I. The Effect of Arthroscopic Surgical Lysis and Lavage of the Superior Joint Space on TMJ Disc Position and Mobility. J Oral Maxillofac Surg. 1989; 47:674-8. 